\title{
Towards a Vorticity Preserving Second Order Finite Volume Scheme Solving the Euler Equations
}

\author{
Farzad Ismail* and Philip L. Roe ${ }^{\dagger}$ \\ Department of Aerospace Engineering-University of Michigan, Ann Arbor, MI 48109-2140, USA
}

In this paper, a second order vorticity preserving finite volume scheme is introduced solving both irrotational and rotational flows. Preserving vorticity requires correcting the numerical fluxes of the momentum equations, so that the curl of the momentum agrees with an independent estimate. The modification does not destroy conservation. The Hancock scheme is used for time-integration with Roe's approximate Riemann solver as the flux function. Preliminary results for the Euler equations in two dimensions indicate that the correction greatly improves the predicted vorticity.

\section{Nomenclature}

U Conservative variables-vector

W Primitive variables-vector

F, G Flux vectors

$\tilde{\mathbf{F}}, \tilde{\mathbf{G}}$ Modified Flux vectors

$\mathbf{P}, \mathbf{Q}$ Flux Correctional vectors

$L_{h} \quad$ Discrete curl Operator

$\omega \quad$ Curl of Velocity $=\nabla \times \vec{u}$

$\Omega \quad$ Curl of Momentum $=\nabla \times(\rho \vec{u})$

$\Phi \quad$ Divergence of Momentum $=\nabla \cdot(\rho \vec{u})$

$\mu_{y} \delta_{x}$ Compact Differencing in x-direction

$\mu_{x} \delta_{y}$ Compact Differencing in y-direction

$\Delta \Omega$ Curl of Momentum Discrepancy

$\Delta t, h$ Time Step and Uniform Mesh Size

Subscript

$i, j \quad$ Variable Cell Coordinates

Superscript

$n \quad$ Discrete Time Level

$k \quad$ Subiteration Time Level

\section{Introduction}

Most successful schemes in Computational Fluid Dynamics (CFD) are based on one dimensional analysis. It is remarkable that these simple one-dimensional ideas which focus on advection and some form of wave

*PhD Candidate and AIAA Student Member.

†Professor and AIAA Fellow. 
propagation can be successfully extended to higher dimensions. However, there are many exceptions where the physics and numerics in one dimension fail to mimic multi-dimensional fluid behavior and vorticity is one of them. In aerospace engineering, there are many problems requiring accurate prediction of vorticity, such as helicopter analysis, and the behavior of high-lift systems, where most schemes will dissipate vorticity excessively. There is also the so-called carbuncle phenomenon where spurious vorticity is generated. In either case, vorticity errors arise out of truncation error in the discretized momentum equations. They are about an order of magnitude smaller than the accuracy of a scheme ${ }^{6}$ but may become significant by accumulation over time. Higher order methods which are costly and perhaps complicated can be used to reduce spurious vorticity.

An alternative is to modify the existing low-order but robust finite volume schemes to be 'vorticity preserving'. By this we mean that vorticity should only be generated by the proper physical mechanisms described by the vorticity transport equation. In particular, if no mechanism for generating vorticity exists, no vorticity should be generated. Conventional cell-centered finite volume schemes based on one-dimensional Riemann problems cannot be vorticity preserving. ${ }^{6}$ This paper will introduce a method that modifies the numerical fluxes of the momentum equations to preserve vorticity to within an error tolerance ${ }^{\mathrm{a}}$. The algorithm utilizes a conventional Roe solver with extra terms to remove spurious vorticity. It is assumed that an independent, and more accurate prediction of vorticity is available, that is from a solution of the vorticity transport equation. Because the underlying scheme is consistent with the Euler equations, the vorticity that it predicts must be, at each time step, close to the true vorticity, and so the corrections that are made must be small. They preserve the formal accuracy of the basic scheme, but prevent the small errors from accumulating into major discrepancies.

The numerical fluxes for the momentum equations are conservatively augmented by small artificial terms before each full step. These terms are obtained by solving an elliptic (Poisson) problem, driven by the discrepancy between the vorticity that would be predicted without any correction, and the vorticity obtained independently from the (pseudo) vorticity transport equation. The Hancock scheme is chosen for timeintegration although the algorithm could be utilized with any other method.

To demonstrate the concept we will utilize a finite volume scheme with Roe-flux differencing on a uniform two-dimensional Cartesian grid. All the conservative and primitive variables are stored in the cell center except for pseudo-vorticity which is defined from the cell quantities as

$$
\Omega=L_{h} \mathbf{U}=\frac{1}{h}\left[\mu_{y} \delta_{x}(\rho v)-\mu_{x} \delta_{y}(\rho u)\right]
$$

and therefore located at the cell vertex (Fig 1). Before we begin with the vorticity preserving analysis, recall that the two-dimensional Euler equations can be written as

$$
\mathbf{U}_{t}+\mathbf{F}(\mathbf{U})_{x}+\mathbf{G}(\mathbf{U})_{y}=0
$$

where $\mathbf{U}=\mathbf{U}(x, y, t), \mathbf{F}(\mathbf{U}), \mathbf{G}(\mathbf{U})$ are determined by

$$
\begin{aligned}
\mathbf{U} & =[\rho, \rho u, \rho v, \rho E]^{T} \\
\mathbf{F}(\mathbf{U}) & =\left[\rho u, \rho u^{2}+P, \rho u v, \rho u H\right]^{T} \\
\mathbf{G}(\mathbf{U}) & =\left[\rho v, \rho u v, \rho v^{2}+P, \rho v H\right]^{T}
\end{aligned}
$$

from the fluid density $\rho$, velocity in $\mathrm{x}$ and $\mathrm{y}$-direction $\mathrm{u}$ and $\mathrm{v}$, the total energy $E$ and the total enthalpy $H=E+\frac{P}{\rho}$. The pressure is determined from the equation of state, which is assumed to be that an ideal gas with constant $\gamma$. Our analysis is presented in two dimensions as a foundation to extend to three dimensions.

\footnotetext{
${ }^{a}$ Strictly, it is the curl of the momentum that is preserved, because that objective makes it easier to retain conservation in the conventional sense. Because there seems to be no generally-accepted term for this quantity, we frequently refer to it loosely as 'vorticity'; when we need to stress the distinction we call it 'pseudo-vorticity'
} 


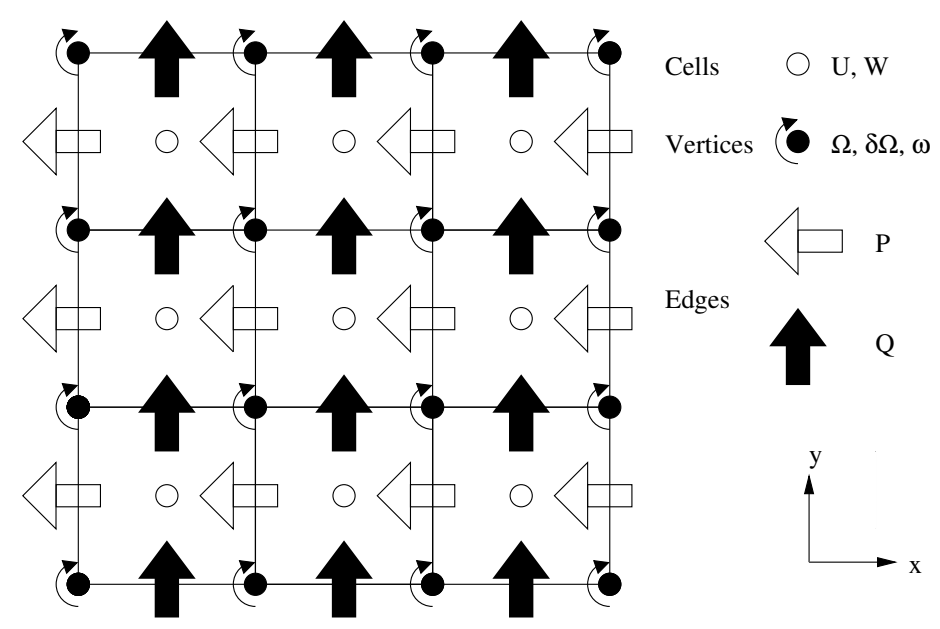

Figure 1. Grid Representation

\section{Preserving Curl of the Momentum}

We intend to alter only the momentum equations since vorticity is hidden in them and leave the mass and energy equations untouched. This is done by adding variable coefficients (or flux corrections) $\mathbf{P}$ and $\mathbf{Q}$ to the numerical fluxes of the momentum equations. Since the order of magnitude of spurious vorticity is smaller than the scheme's order of accuracy, we will introduce small corrections to the momentum equations. Recall that the approximate Riemann problem with interface separated by a left and right state solved by Roe-solver in one-dimension can be expressed as

$$
\mathbf{F}_{I}=\frac{1}{2}\left[\mathbf{F}_{L(B)}+\mathbf{F}_{R(T)}\right]-\sum_{k}|\lambda| \Delta \mathbf{V}_{k} \mathbf{r}_{k}
$$

where $\lambda$ are the wave speeds and $\Delta \mathbf{V}$ with $\mathbf{r}_{k}$ are the wave amplitudes and right eigenvectors respectively. To preserve vorticity, we alter the x-momentum by a small correction in the y-direction $q$ and likewise the $\mathrm{y}$-momentum by $p$ in the $\mathrm{x}$-direction. Our modified fluxes at the interface will have the following form.

$$
\begin{aligned}
\tilde{\mathbf{F}}_{I} & =\frac{1}{2}\left[\mathbf{F}\left(\mathbf{U}_{L}\right)+\mathbf{F}\left(\mathbf{U}_{R}\right)\right]-\sum_{k}\left(|\lambda| \Delta \mathbf{V}_{k} \mathbf{r}_{k}\right)-\mathbf{P} \\
\tilde{\mathbf{G}}_{I} & =\frac{1}{2}\left[\mathbf{G}\left(\mathbf{U}_{B}\right)+\mathbf{G}\left(\mathbf{U}_{T}\right)\right]-\sum_{k}\left(|\lambda| \Delta \mathbf{V}_{k} \mathbf{r}_{k}\right)-\mathbf{Q}
\end{aligned}
$$

where we introduce the interface flux corrections $\mathbf{P}$ and $\mathbf{Q}$ only to the momentum equations given by

$$
\begin{aligned}
& \mathbf{P}=[0,0, p, 0]^{T} \\
& \mathbf{Q}=[0, q, 0,0]^{T}
\end{aligned}
$$

Hence we shall discretize the two-dimensional Euler equations using the standard and modified fluxes i.e.

$$
\begin{aligned}
& \mathbf{U}_{i, j}^{n+1}=\mathbf{U}_{i, j}^{n}-\frac{\Delta t}{h}\left[\mathbf{F}_{i+\frac{1}{2}, j}^{n}-\mathbf{F}_{i-\frac{1}{2}, j}^{n}+\mathbf{G}_{i, j+\frac{1}{2}}^{n}-\mathbf{G}_{i, j-\frac{1}{2}}^{n}\right] \\
& \tilde{\mathbf{U}}_{i, j}^{n+1}=\tilde{\mathbf{U}}_{i, j}^{n}-\frac{\Delta t}{h}\left[\tilde{\mathbf{F}}_{i+\frac{1}{2}, j}^{n}-\tilde{\mathbf{F}}_{i-\frac{1}{2}, j}^{n}+\tilde{\mathbf{G}}_{i, j+\frac{1}{2}}^{n}-\tilde{\mathbf{G}}_{i, j-\frac{1}{2}}^{n}\right]
\end{aligned}
$$


where $\mathbf{U}_{i, j}^{n}$ and $\tilde{\mathbf{U}}_{i, j}^{n}$ are the standard and modified cell averages of the conservative variables at coordinate $(\mathrm{i}, \mathrm{j})$ and time level $\mathrm{n}$. We define pseudo-vorticity $\Omega$ at a vertex through the compact difference of four neighboring cell-center momenta $(\rho \vec{u})$ that surround the vertex, as in (1). Controlling this particular discretization of the vorticity allows the checkerboard mode of the momenta in the cells, although not in the fluxes. There are other ways to define discrete vorticity using larger stencils but these come with even more spurious modes. ${ }^{6}$ The corrected and uncorrected vorticities are related by

$$
\tilde{\Omega}^{n+1}=\Omega^{n+1}+\frac{\Delta t}{h^{2}}\left[\mu_{y} \delta_{x}^{2} p-\mu_{x} \delta_{y}^{2} q\right]^{n}
$$

We will insist that the corrected vorticity is identical to some independent estimate that will be discussed in the following section,

$$
\tilde{\Omega}^{n+1}=\Omega_{I N D}^{n+1}
$$

and to achieve this we will use subiterations within the timestep. Denote conditions after the $k$ th subiteration by ()$^{k}$ and conditions after the subiterations have converged by ()$^{\infty}$. The problem that we wish to solve is

$$
\Omega^{\infty}=\Omega_{I N D}^{n+1}=\Omega^{n+1}+\frac{\Delta t}{h^{2}}\left[\mu_{y} \delta_{x}^{2} p^{\infty}-\mu_{x} \delta_{y}^{2} q^{\infty}\right]
$$

where $p^{\infty}, q^{\infty}$ are the corrections that need to be determined. The sub-iterations that bring this about are

$$
\Omega^{k+1}=\Omega^{k}+\frac{\Delta t}{h^{2}}\left[\mu_{y} \delta_{x}^{2}\left(p^{k+1}-p^{k}\right)-\mu_{x} \delta_{y}^{2}\left(q^{k+1}-q^{k}\right)\right]
$$

where we assume $\Omega^{n+1}$ as the initial values for the subiterations. The particular choices

$$
\begin{aligned}
& p^{k+1}-p^{k}=\zeta \mu_{y}\left(\Omega^{k}-\Omega_{I N D}^{n+1}\right) \\
& q^{k+1}-q^{k}=-\zeta \mu_{x}\left(\Omega^{k}-\Omega_{I N D}^{n+1}\right)
\end{aligned}
$$

with $\zeta$ as a parameter that restricts the relaxation factor will lead to the discrete Laplacian operator

$$
\Omega^{k+1}=\Omega^{k}+\kappa\left[\mu_{y}^{2} \delta_{x}^{2}\left(\Omega^{k}-\Omega_{I N D}^{n+1}\right)+\mu_{x}^{2} \delta_{y}^{2}\left(\Omega^{k}-\Omega_{I N D}^{n+1}\right)\right]
$$

where $\kappa=\zeta \frac{\Delta t}{h^{2}}$ is the relaxation. Define vorticity discrepancy as

$$
\Delta \Omega^{k}=\Omega^{k}-\Omega_{I N D}^{n+1}
$$

so that

$$
\Delta \Omega^{k+1}=\Delta \Omega^{k}+\kappa\left[\mu_{y}^{2} \delta_{x}^{2}+\mu_{x}^{2} \delta_{y}^{2}\right] \Delta \Omega^{k}
$$

which is an elliptic relaxation for $\Delta \Omega$. Clearly this problem has no intrinsic interest because its steady solution is identically zero. It is however, the mechanism by which $p$ and $q$ are generated in equation (15). Incorporating these flux corrections preserves conservation of momentum, but redistributes it so as to be compatible with the independent calculation.

Note that this discrete Laplacian of the vorticity uses a bad stencil, the rotated $\mu_{y}^{2} \delta_{x}^{2}+\mu_{x}^{2} \delta_{y}^{2}$ rather than the standard five point $\delta_{x}^{2}+\delta_{y}^{2}$. This introduces odd-even decoupling into the vorticity, but when this is averaged to give $p$ and $q$ the spurious mode disappears. 


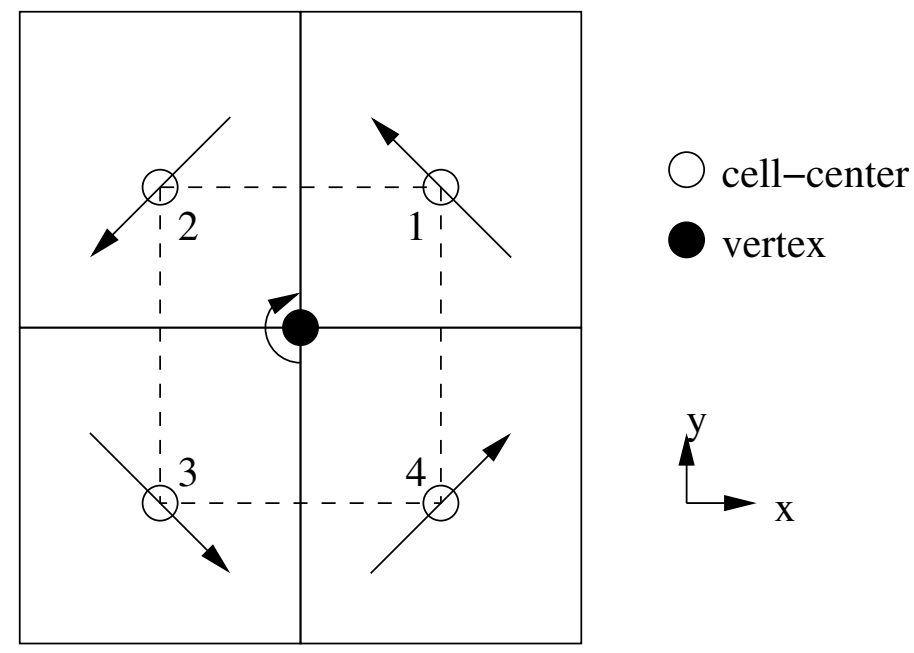

Figure 2. Removing spurious vorticity with flux-correction. Assume we have a clockwise spurious vorticity $\Delta \Omega^{n+1}$ at a vertex, we introduce a counter-clockwise correction altering the momenta. Note that this correction does not change the discrete divergence of momentum within the vertex control volume.

\section{Independent Estimate of Vorticity}

We shall derive an integral form of vorticity conservation by taking the curl of the momentum equations. for an arbitrary control volume $d V$ with surface $S$, the inviscid momentum equations can be written in the following form

$$
\frac{\partial}{\partial t} \iiint_{V} \rho \vec{U} d V+\oint_{S}(\rho \vec{U} \cdot \overrightarrow{d S}) \vec{U}+\oint_{S} P \overrightarrow{d S}=0
$$

Appealing to the Divergence Theorem, we can rewrite the last two terms as

$$
\begin{aligned}
\oint_{S}(\rho \vec{U} \cdot \overrightarrow{d S}) \vec{U} & =\iiint_{V} \nabla \cdot(\rho \vec{U} \vec{U}) d V \\
\oint_{S} P \overrightarrow{d S} & =\iiint_{V} \nabla P d V
\end{aligned}
$$

We define vorticity as $\vec{\Omega}=\nabla \times \rho \vec{U}$ and applying the curl operator on the momentum equations we get

$$
\frac{\partial}{\partial t} \iiint_{V} \vec{\Omega} d V+\iiint_{V} \nabla \times[\nabla \cdot(\rho \vec{U} \vec{U})] d V=0
$$

since the $\nabla \times \nabla$ operator is zero. Recall that the dyadic tensor is defined as

$$
\vec{U} \vec{U}=\vec{U} \circ \vec{U}=\left[\begin{array}{ccc}
u^{2} & u v & u w \\
v u & v^{2} & v w \\
w u & w v & w^{2}
\end{array}\right]
$$


Note that $\rho \vec{U} \circ \vec{U}=\vec{U} \circ \rho \vec{U}$. Also, recall the following vector identities.

$$
\begin{aligned}
& \nabla \cdot(\vec{A} \circ \vec{B})=\vec{B} \circ \nabla \vec{A}+\vec{A}(\nabla \cdot \vec{B}) \\
& \vec{A} \times \nabla \times \vec{B}=\frac{1}{2} \nabla(\vec{A} \cdot \vec{B})-\vec{B} \circ \nabla \vec{A} \\
& \nabla \times \vec{A} \times \vec{B}=\vec{A} \nabla \cdot \vec{B}-\vec{B} \nabla \cdot \vec{A}+(\vec{B} \cdot \nabla) \vec{A}-(\vec{A} \cdot \nabla) \vec{B}
\end{aligned}
$$

Using the first identity we expand the divergence term as

$$
\nabla \cdot(\vec{U} \circ \rho \vec{U})=\rho \vec{U} \circ \nabla \vec{U}+\vec{U}(\nabla \cdot \rho \vec{U})
$$

Using the second vector identity we write the first term of the previous equation as

$$
\rho \vec{U} \circ \nabla \vec{U}=\frac{1}{2} \nabla(\vec{U} \cdot \rho \vec{U})-\vec{U} \times \nabla \times \rho \vec{U}
$$

The curl of this equation is

$$
\begin{aligned}
\nabla \times[\vec{U} \circ \nabla \rho \vec{U}] & =\nabla \times\left[\frac{1}{2} \nabla(\rho \vec{U} \cdot \vec{U})\right]-\nabla \times[\vec{U} \times \nabla \times \rho \vec{U}] \\
& =-\nabla \times[\vec{U} \times \vec{\Omega}] \\
& =(\vec{U} \cdot \nabla) \vec{\Omega}+\vec{\Omega} \nabla \cdot \vec{U}-(\vec{\Omega} \cdot \nabla) \vec{U} \\
& =\nabla \cdot(\vec{\Omega} \circ \vec{U})-(\vec{\Omega} \cdot \nabla) \vec{U}
\end{aligned}
$$

after using the third vector identity and that $\nabla \cdot \nabla \times$ is trivial. All together, the curl of momentum for an arbitrary control volume $\mathrm{V}$ can be written as

$$
\iiint_{V}\left[\frac{\partial \vec{\Omega}}{\partial t}+\nabla \cdot(\vec{\Omega} \circ \vec{U})-(\vec{\Omega} \cdot \nabla) \vec{U}+\nabla \times[\vec{U}(\Phi)]\right] d V=0
$$

The first three terms correspond to unsteady vorticity, vorticity advection and vortex stretching. The last term represents compressibility effects. Note for incompressible flow, we can rewrite the equation as

$$
\iiint_{V}\left[\frac{\partial \vec{\omega}}{\partial t}+\nabla \cdot(\vec{\omega} \circ \vec{U})-(\vec{\omega} \cdot \nabla) \vec{U}\right] d V=0
$$

recovering the conventional inviscid vorticity evolution equation given in. ${ }^{2}$

\section{A. Two-Dimensional Case}

In two-dimensions, $\vec{\Omega}=\left[0,0, \Omega_{z}\right]^{T}$ hence the vortex stretching term drops out and

$$
\begin{aligned}
& \nabla \cdot(\vec{\Omega} \circ \vec{U})=\frac{\partial\left(u \Omega_{z}\right)}{\partial x}+\frac{\partial\left(v \Omega_{z}\right)}{\partial y} \\
& \nabla \times[\vec{U}(\Phi)]=\frac{\partial(v \Phi)}{\partial x}-\frac{\partial(u \Phi)}{\partial y}
\end{aligned}
$$

Define normal velocity vectors $\vec{U}_{n}=[v,-u]^{T}$ thus vorticity evolution equation becomes

$$
\iint_{S}\left[\frac{\partial \Omega_{z}}{\partial t}+\nabla \cdot\left(\vec{U} \Omega_{z}+\vec{U}_{n} \Phi\right)\right] d S=0
$$

$$
6 \text { of } 14
$$


This equation is in conservation form ${ }^{\mathrm{b}}$. Applying the Divergence Theorem we get

$$
\iint_{S} \frac{\partial \Omega_{z}}{\partial t} d S+\oint_{l}\left(\vec{U} \Omega_{z}+\overrightarrow{U_{n}} \Phi\right) \cdot \overrightarrow{d l}=0
$$

where $S$ is an arbitrary area closed by a line $l$. For brevity, we will assume from now on that $\Omega_{z}=\Omega$. In three dimensions, conservation form is lost due to the stretching term.

\section{B. Discretization in 2D}

Discrete conservation is ensured by drawing a control volume around the point of interest (in this case it is the vertex) and perform the update as an integral around the control volume. Appealing to a uniform Cartesian grid, with fluxes $\mathrm{F}, \mathrm{G}$ in the $(\mathrm{x}, \mathrm{y})$-directions, respectively, the vorticity at the vertices are discretized as

$$
h^{2}\left[\Omega^{n+1}-\Omega^{n}\right]+h \Delta t\left[\delta_{x} F_{*}+\delta_{y} G_{*}\right]=0
$$

where $F_{*}, G_{*}$ are numerical fluxes evaluated from some formula to be determined. We shall appeal to staggered grid formulation defined in Fig. 3. The primitive variables are located in the cell-center but vorticity is located at the vertices. We need the velocities (both normal and tangential velocities) at the interface of the control volume that defines vorticity. We have the primitive values at the cell-centers which encloses the control volume of vorticity. At any interface, the primitive values are the average of two primitive cell-centered values. In two dimensions there are two terms in the equation for vorticity evolution: the gradients of pseudo-vorticity and momentum divergence. We chose to treat the former as an advection term to be upwinded. The latter is discretized using a central scheme since it is a divergence of a gradient quantity and is diffusion-like. We will evaluate the interface fluxes as the following.

$$
\begin{aligned}
F_{*} & =\left[u f_{u p}\left(\Omega_{L}, \Omega_{R}\right)+v \frac{1}{2}\left((\Phi)_{L}+(\Phi)_{R}\right)\right] \\
G_{*} & =\left[v f_{u p}\left(\Omega_{B}, \Omega_{T}\right)-u \frac{1}{2}\left((\Phi)_{B}+(\Phi)_{T}\right)\right]
\end{aligned}
$$

where $u, v$ indicate interface velocities and $\Phi$ is the momentum divergence located at the vertices evaluated via compact divergence utilizing cell-centered velocities.

$$
\Phi=\frac{1}{h}\left[\mu_{y} \delta_{x}(\rho u)+\mu_{x} \delta_{y}(\rho v)\right]
$$

The one-dimensional upwinded horizontal flux function is evaluated as

$$
\begin{aligned}
& f_{u p}\left(\Omega_{L}, \Omega_{R}\right)=\Omega_{L}(u>0) \\
& f_{u p}\left(\Omega_{L}, \Omega_{R}\right)=\Omega_{R}(u<0)
\end{aligned}
$$

Note if the interface velocity is zero, the flux will be an average of the two states. The vertical fluxes are evaluated by a similar process. With first order forward Euler time integration method, the independent estimate for vorticity is updated as

$$
\Omega_{I N D}^{n+1}=\Omega_{I N D}^{n}-\frac{\Delta t}{h}\left[\delta_{x} F_{*}+\delta_{y} G_{*}\right]
$$

\footnotetext{
${ }^{\mathrm{b}}$ This does not mean that pseudo-vorticity is a conserved quantity, unless it also obeys suitable jump conditions
} 


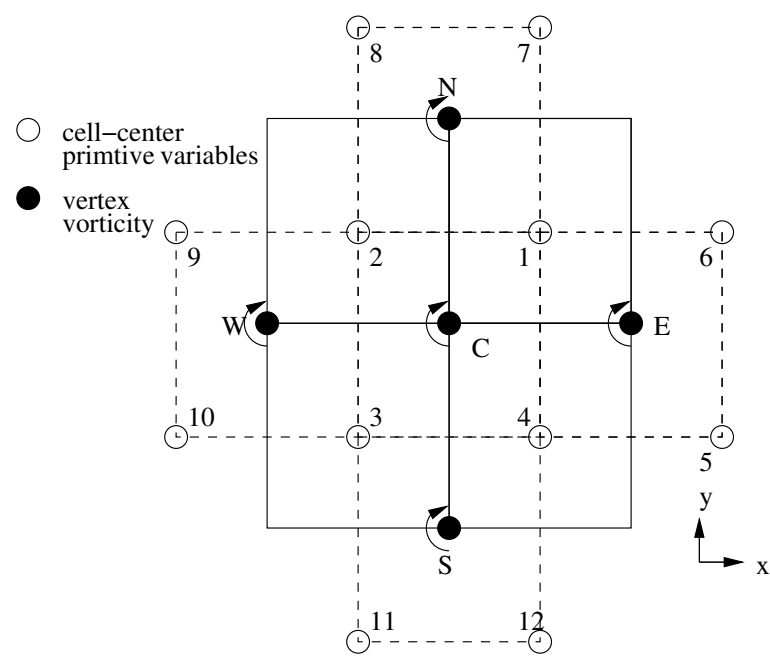

Figure 3. Control volume for vorticity is marked by dashed-lines. For a particular CV with centroid C, the momentum divergence at the vorticity interface is an averaged-differencing utilizing vertices [C,E] for interface $(1,4) ;[C, N]$ for for interface $(1,2) ;[C, W]$ for interface $(2,3) ;[C, S]$ for for interface $(3,4)$. For vorticity advection, each interface is governed by pairs for upwinding, also denoted as $[\mathrm{C}, \mathrm{E}],[\mathrm{C}, \mathrm{N}],[\mathrm{C}, \mathrm{W}]$ and $[\mathrm{C}, \mathrm{S}]$. The interface velocities are just the averaged of velocties at $(1,4),(1,2),(2,3)$ and $(3,4)$.

\section{Second Order Method}

We will now construct a second order scheme for vorticity evolution. The time-integration will be second order Runge-Kutta with advection components of the numerical fluxes being linearly reconstructed. To avoid spurious overshoots, we will limit these fluxes. The divergence of momentum will still be discretized as a central scheme. This will be a two-step scheme in which we have two options. The first is where limiter is applied to the half-step only (VP1) and the other is where limiter is applied to both half and full-steps (VP2). The slope limiting is based on one-dimensional considerations hence any conventional one-dimensional limiter can be used, and we have chosen the Superbee limiter. Since our grids are uniform, we can define the limited slopes of vorticity in $\mathrm{x}$ and $\mathrm{y}$-directions centered at the vertices as

$$
\begin{aligned}
& \delta \Omega_{i, j}^{x}=\lim \left(\Omega_{i, j}-\Omega_{i-1, j}, \Omega_{i+1, j}-\Omega_{i, j}\right) \\
& \delta \Omega_{i, j}^{y}=\lim \left(\Omega_{i, j}-\Omega_{i, j-1}, \Omega_{i, j+1}-\Omega_{i, j}\right)
\end{aligned}
$$

To obtain second order spatial accuracy, we shall use these limited slopes to reconstruct the left and right states of the cell-vertex scheme.

$$
\begin{aligned}
& \tilde{\Omega}_{L}=\Omega_{L}+\frac{1}{2} \delta \Omega_{L}^{x} \\
& \tilde{\Omega}_{R}=\Omega_{R}-\frac{1}{2} \delta \Omega_{R}^{x}
\end{aligned}
$$

In the vertical direction, a similar method is used. These values will be used in the upwinded function defined on the previous section thus the limited fluxes are evaluated as

$$
\begin{aligned}
& \tilde{F}_{*}=\left[u f_{u p}\left(\tilde{\Omega}_{L}, \tilde{\Omega}_{R}\right)+v \frac{1}{2}\left((\Phi)_{L}+(\Phi)_{R}\right)\right] \\
& \tilde{G}_{*}=\left[v f_{u p}\left(\tilde{\Omega}_{B}, \tilde{\Omega}_{T}\right)-u \frac{1}{2}\left((\Phi)_{B}+(\Phi)_{T}\right)\right]
\end{aligned}
$$


The half and full-steps for the vorticity preserving scheme (VP1) are written as

$$
\begin{aligned}
& \Omega_{I N D}^{n+\frac{1}{2}}=\Omega_{I N D}^{n}-\frac{\Delta t}{h}\left[\delta_{x} \tilde{F}_{*}^{n}+\delta_{y} \tilde{G}_{*}^{n}\right] \\
& \Omega_{I N D}^{n+1}=\Omega_{I N D}^{n}-\frac{\Delta t}{h}\left[\delta_{x} F_{*}^{n+\frac{1}{2}}+\delta_{y} G_{*}^{n+\frac{1}{2}}\right]
\end{aligned}
$$

where the full-step fluxes are identical to fluxes derived in the previous section. For VP2 scheme, we update the full-step with linearly reconstructed and limited fluxes.

\section{Algorithm}

There will be two distinct numerical processes, one solving the Euler equations while the other solves the vorticity evolution. The former is computed via Hancock scheme with Superbee limiter while the latter will be evaluated using the discretized scheme discussed in the previous section. Both will be coupled and run concurrently with each other. The algorithm below describes the steps involved.

- Compute vorticity estimate at time level $\mathrm{n}+1\left(\Omega_{I N D}^{n+1}\right)$ using equation (49) with variables at time $\mathrm{n}$.

- Compute the Euler equations using Hancock scheme. Solve the conventional 1D Riemann problem using Roe-solver and hence a provisional solution, with provisional vorticity $\Omega^{n+1}$ and

1. Compute initial vorticity discrepancy, $\Delta \Omega^{0}=\Omega^{n+1}-\Omega_{I N D}^{n+1}$ at each vertex with initial $p=q=0$ and $\Omega^{0}=\Omega^{n+1}$.

2. Solve the Poisson equation for $\Delta \Omega^{k+1}$ using a relaxation scheme with $\Delta \Omega=0$ at the boundaries for one sub-iteration step.

3. Perform correction on $p$ and $q$ at each cell-interface based on the updated values $\Delta \Omega^{k+1}$ using equation (15).

4. Compute the updated vorticity discrepancy, if $\Delta \Omega^{k+1}<$ error tolerance, stop the iteration and compute the overall fluxes at each interface and update the conservative variables at time level $\mathrm{n}+1$. Else go back to step 2 .

- Compute other variables such as primitive variables and compact vorticity at time $n+1$. To compute the next time-level, start again from the beginning.

\section{Results}

We can apply the method with particular ease to situations where we know in advance that no vorticity is generated. One such case is steady flow through a plane shock. This trivial flow poses problems for many numerical methods because a vortical mode proves unstable, leading to the 'carbuncle phenomenon'. We have chosen the initial conditions proposed by Dumbser et. al. ${ }^{3}$ as our test for preserving irrotationality. Of course the independent computation of vorticity is not required in this case.

In general, vorticity is produced by both viscous and inviscid mechanisms. We want to check whether its subsequent transport by an inviscid flow will be correct. For rotational flow, we came up with a test problem that features vorticity advected in a square domain with periodic boundary conditions. We will set Courant number $\nu=0.8$ (Carbuncle) and $\nu=0.9$ (Vorticity Advection) with $\zeta=0.6$ to restrict the relaxation parameter $\kappa$. To solve the Poisson problem given by equation (18), we have used the Gauss-Seidel method. Clearly a better relaxation scheme should speed the method up considerably. 


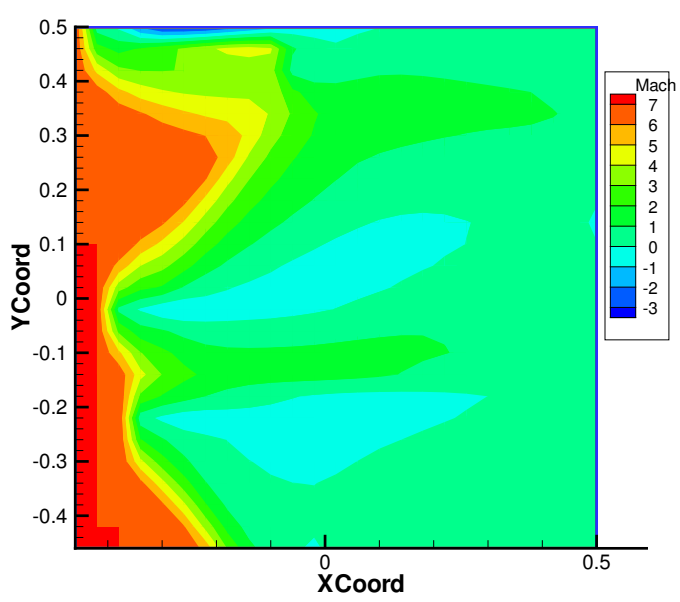

Figure 4. First order Upwind at $\mathbf{T}=1000$

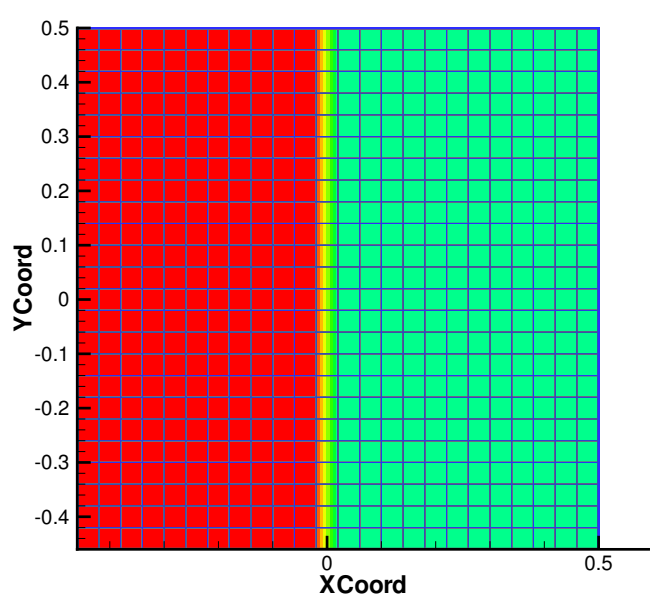

Figure 5. VP with Superbee at $T=10000$

\section{A. Carbuncle}

This is a test problem which produces shock instabilities, leading to vorticity. It is a simplified carbuncle problem described by a steady normal shock wave computed on two-dimensional uniform Cartesian grids. The initial and boundary conditions are identical to, ${ }^{3}$ except that we assumed the outflow (right) to leave the domain by setting zero slopes in all ghost cells and for all conservative variables. This carbuncle problem may be driven by small perturbations to the variables upstream of the shock, or it may arise spontaneously without being provoked. We employed very small perturbations, of order $10^{-16}$.

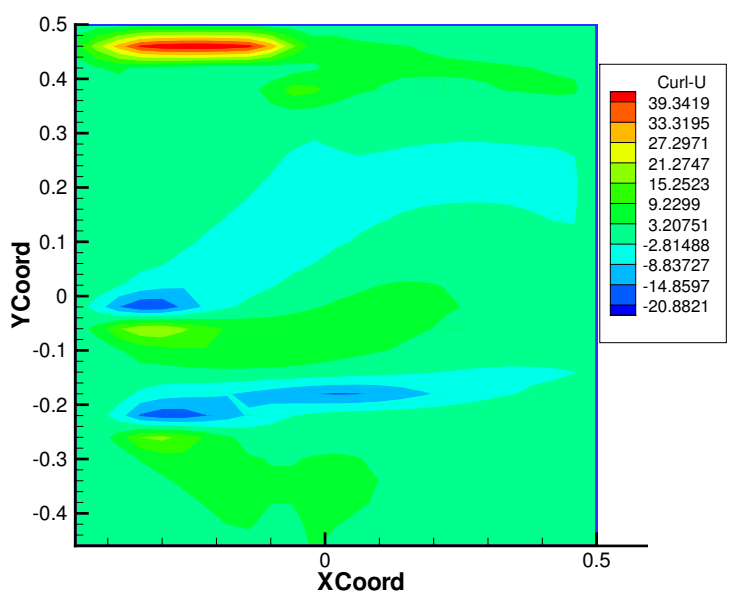

Figure 6. Vorticity (1st order Upwind) at $\mathbf{T}=1000$

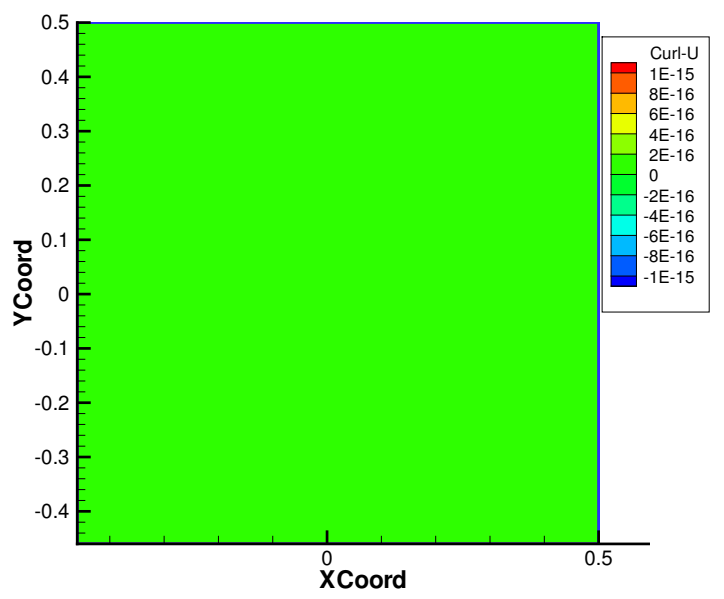

Figure 7. Vorticity (VP-Superbee) at $\mathbf{T}=10000$ 


\section{Rankine-Hugoniot Jump Conditions}

Assuming $M_{0}$ is the upstream Mach number with $\gamma=1.4$, the normalized upstream and downstream conditions are given by

$$
\begin{aligned}
& \mathbf{U}_{0}=\left[\begin{array}{llll}
1 & 1 & 0 & \frac{1}{\gamma(\gamma-1) M_{0}^{2}}+\frac{1}{2}
\end{array}\right] \\
& \mathbf{U}_{1}=\left[\begin{array}{llll}
f\left(M_{0}\right) & 1 & 0 & \frac{g\left(M_{0}\right)}{\gamma(\gamma-1) M_{0}^{2}}+\frac{1}{2 f\left(M_{0}\right)}
\end{array}\right]
\end{aligned}
$$

where $f\left(M_{0}\right)$ and $g\left(M_{0}\right)$ are the jump conditions of density and pressure across the shock given by

$$
\begin{aligned}
& f\left(M_{0}\right)=\left(\frac{2}{(\gamma+1) M_{0}^{2}}+\frac{\gamma-1}{\gamma+1}\right)^{-1} \\
& g\left(M_{0}\right)=\frac{2 \gamma M_{0}^{2}}{(\gamma+1)}-\frac{\gamma-1}{\gamma+1}
\end{aligned}
$$

Our results indicate that for a Mach 7 flow, the vorticity preserving Hancock scheme (VP) maintains the shock structure (Fig 5) unlike the first order Hancock scheme (Fig 4). This is for a vorticity discrepancy tolerance of $10^{-16}$ consuming about 80 iterations per time step. Indeed, the VP scheme preserves irrotationality even after 10000 time-steps.

\section{B. Vorticity Advection}

This is to simulate a simple vorticity advection that satisfies the Euler equations with periodic boundary conditions. We derived a simple exact solution by assuming steady flow with a Gaussian pressure disturbance. Then we found a divergence-free velocity field satisfying $\frac{\partial P}{\partial r}=\frac{\rho \vec{U}^{2}}{r}$, and finally superposed a uniform advection speed. The solution is

$$
\begin{aligned}
& u=M \cos \alpha+c_{1}\left[y-y_{o}-M t \sin \alpha\right] \exp \left[-c_{2}\left(\left[x-x_{o}-M t \cos \alpha\right]^{2}+\left[y-y_{o}-M t \sin \alpha\right]^{2}\right)\right] \\
& v=M \sin \alpha-c_{1}\left[x-x_{o}-M t \cos \alpha\right] \exp \left[-c_{2}\left(\left[x-x_{o}-M t \cos \alpha\right]^{2}+\left[y-y_{o}-M t \sin \alpha\right]^{2}\right)\right] \\
& P=P_{b}-0.25 \rho \frac{c_{1}^{2}}{c_{2}}\left(\exp \left[-2 c_{2}\left(\left[x-x_{o}-M t \cos \alpha\right]^{2}+\left[y-y_{o}-M t \sin \alpha\right]^{2}\right)\right]-1\right)
\end{aligned}
$$

where $M, \alpha$ and $P_{b}$ are the background flow Mach number $(M=0.5)$, flow angle $(\alpha=0)$ and pressure $\left(P_{b}=1.0\right)$ with perturbation coefficients $\left(c_{1}, c_{2}\right)=(-0.04,0.02)$. The reference coordinates are chosen to be $\left(x_{o}, y_{o}\right)=(-20,0)$. For simplicity, we set density to be a constant $(\rho=1.4)$. For better density representation, we could utilize the isentropic relations $\frac{P}{\rho \gamma}=k$ but did not do so in this example.

Our results demonstrate that the VP2 scheme is superior to any other schemes (Fig 13) preserving enstrophy closest to the exact solution even after 450 time-steps. The first order Hancock is the worst, diffussing 60 percent of enstrophy after 100 time-steps. The second order Hancock scheme with Superbee limiter does not diffuse enstrophy due to its nature of steepening slopes when there is no danger of overshoot. This works well with shocks, however, for this case the steepening generates spurious enstrophy with time.

The VP2 scheme also uses Superbee to limit its Euler and vorticity fluxes but only produces negligible amounts of spurious enstrophy. This is attributed to vorticity physics being incorporated to the scheme in addition to conventional upwinding. The performance of the VP1 scheme lies in between the first order upwind and the Superbee method. This is quite unsurprising since vorticity accuracy depends strongly on the discretization of its independent estimate. When solving the vorticity evolution, the VP1 reconstructs a linear flux function only at the half-step with the full-step being spatially first order. This contributes to excess diffusion of enstrophy. In addition, Fig (15) illustrates that for this simple case, solving the Poisson equation with 50 or 2000 iterations yields about 1 percent error for the VP2 scheme.

11 of 14

American Institute of Aeronautics and Astronautics 


\section{Concluding Remarks}

Our results indicate that by controlling the curl of the momentum, we have also controlled the curl of velocity. The vorticity preserving scheme utilizing Roe's flux function does not suffer from the carbuncle phenomenon. This by no means implies that we have solved the carbuncle problem ${ }^{8}$ (generally, the carbuncle occurs in practice ahead of blunt bodies, and the slightly curved bow shock should produce some vorticity) but rather a demonstration that preserving irrotationality can influence its onset.

For rotational flow, preserving vorticity depends heavily on the accuracy of the independent vorticity estimate. The VP2 scheme utilizes limited second order Hancock and linearly reconstruct the numerical fluxes of vorticity evolution at both time-steps hence producing the best overall second order scheme (Fig 16 and Fig 17). As a result of explicitly including the physics of vorticity, not only is the method second order accurate for the primary variables (primitive and conservative variables) but also its derived vorticity is much more accurate than the regular second-order Hancock scheme. Although this generates second order accurate primary variables, our experiments show that it only produces first order accuracy for the vorticity. This is much worse than the theoretical estimate in, ${ }^{6}$ probably due to the error being cumulative.

There is a need to analyze and perhaps improve the convergence rate of solving the Poisson equation. At the moment, the Gauss-Seidel method with $\kappa=0.6$ works quite well for our demonstration. The ultimate goal of this work to extend this method to three dimensions and is currently underway.

\section{References}

\footnotetext{
${ }^{1}$ Anderson, J.D., "Modern Compressible Flow with Historical Perspective, 2nd Edition", pp. 32-43, 1990.

${ }^{2}$ Batchelor, G.K, , "An Introduction to Fluid Dynamics", pp. 265-280, reprinted 2001.

${ }^{3}$ Dumbser, M., Moschetta, J.M., and Gressier, J., "A Matrix Stability Analysis of the Carbuncle Phenomenon", to appear J. Comp. Physics, 2004

${ }^{4}$ Greenberg, M.D, “Advanced Engineering Mathematics", pp. 270-435, 1988.

${ }^{5}$ Lowrie, R.B., "Compact Higher Order Numerical Methods for Hyperbolic Conservation Laws", PhD Thesis, University of Michigan., 1996

${ }^{6}$ Morton, K.W., and Roe, P.L., "Vorticity-Preserving Lax-Wendroff Type Schemes for the System Wave Equation", J. Sci. Comp, Vol. 23, No.1, pp. 170-192, January 2001.

${ }^{7}$ Sanders, R., Morano, E., Druguet, M.C., "Multidimensional Dissipation for Upwind Schemes: Stability and Applications to Gas Dynamics", J. Comp. Physics, Vol. 145, pp. 511-537, 1998.

${ }^{8}$ Roe, P.L, Nishikawa, H., Ismail, F., Scalabrin, L.C, "On Carbuncles and Other Excresences", to appear AIAA 17th CFD Conference-Toronto, Conference Paper-AIAA, 2005.
}

12 of 14 


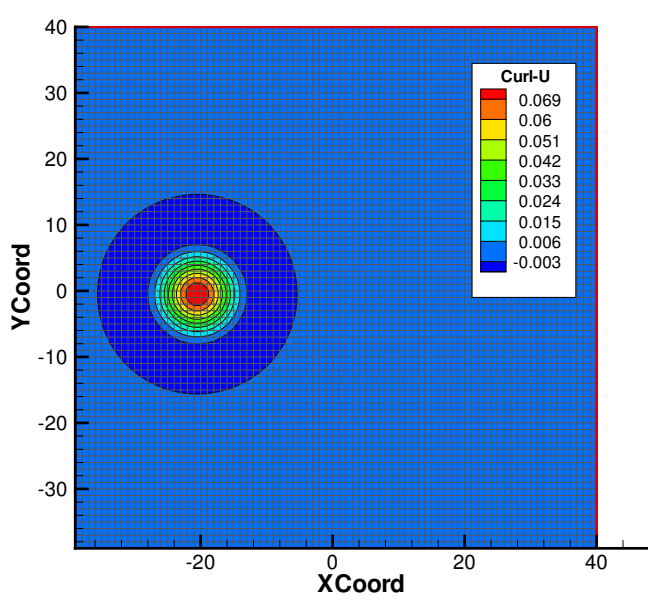

Figure 8 . Vorticity at $T=0$ with $80 \times 80$ cells

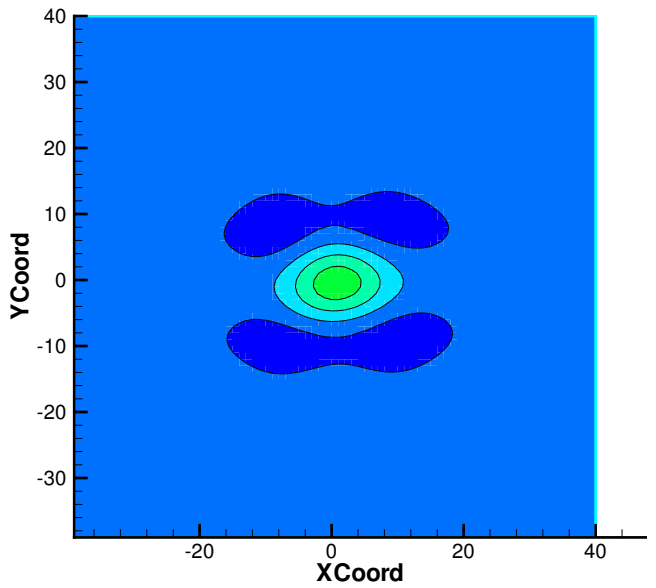

Figure 10. Vorticity (1st order Upwind) at $\mathbf{T}=\mathbf{1 8 0}$

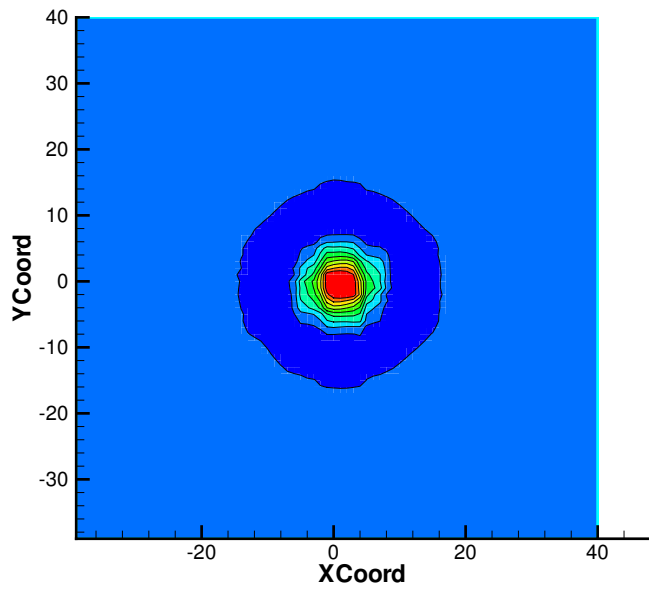

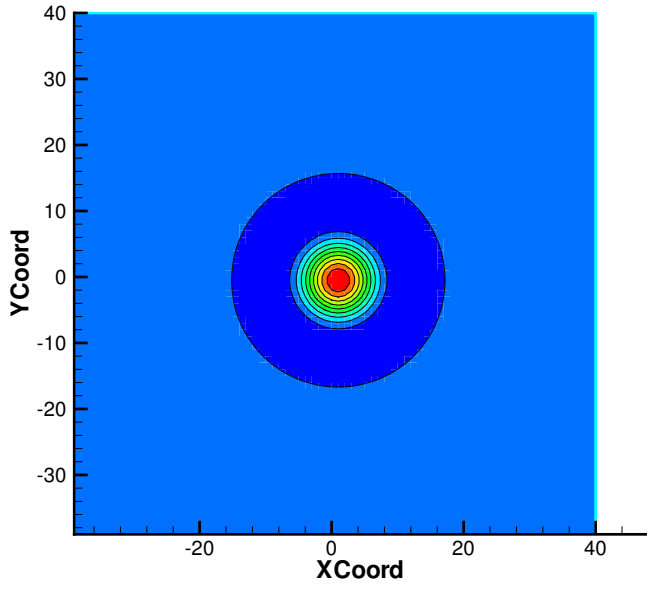

Figure 9. Exact Vorticity at $\mathbf{T}=\mathbf{1 8 0}$

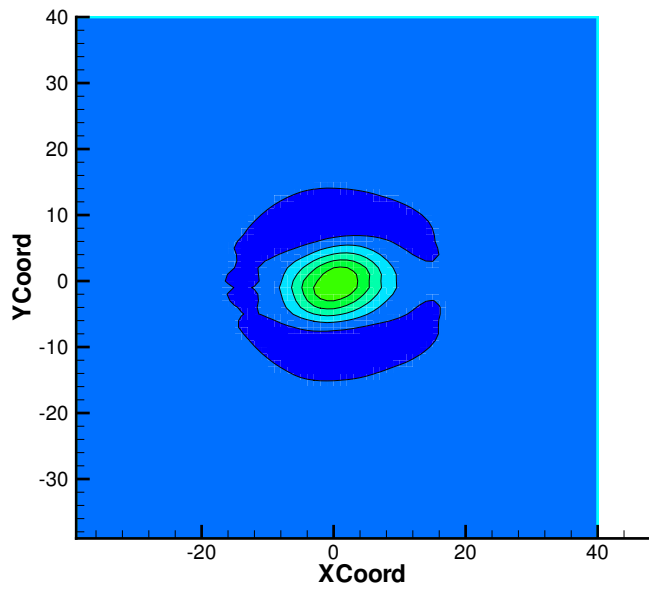

Figure 11. Vorticity (Superbee-VP1) at $\mathbf{T}=\mathbf{1 8 0}$

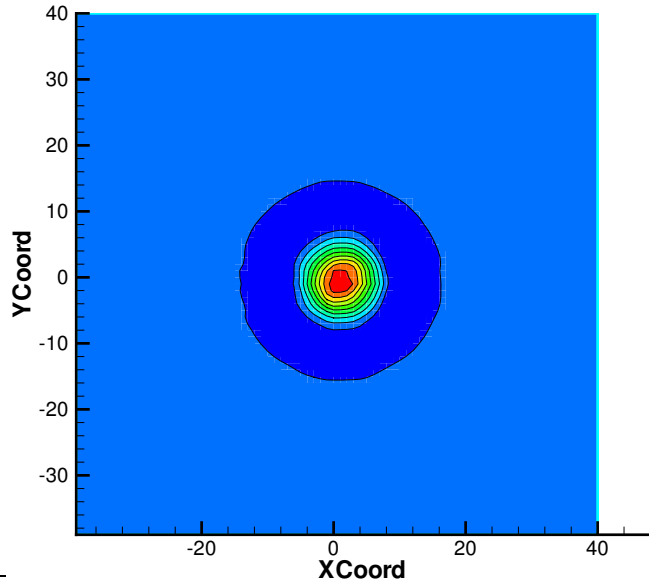

13 of 14

Figure 12. Vorticity (Superbee) at $\mathbf{T}=\mathbf{1 8 0}$

Figure 13. Vorticity (Superbee-VP2) at $\mathbf{T}=\mathbf{1 8 0}$ 


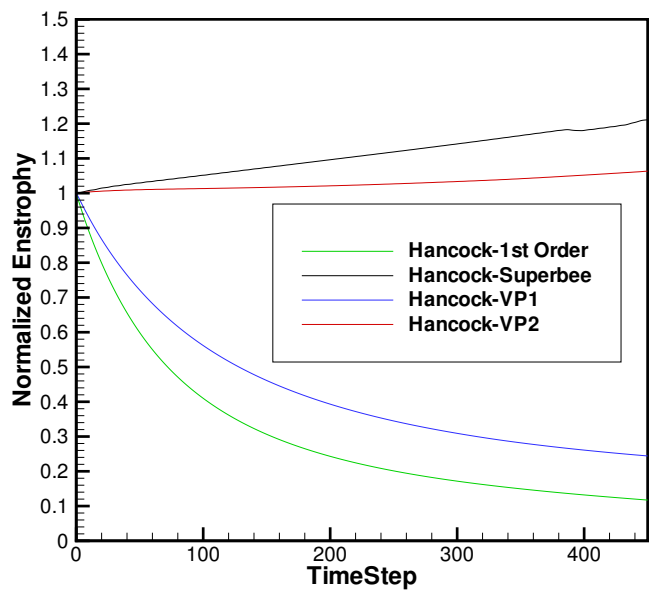

Figure 14. Normalized Enstrophy for various schemes using $80 \times 80$ cells. Note that exact enstrophy is unity.

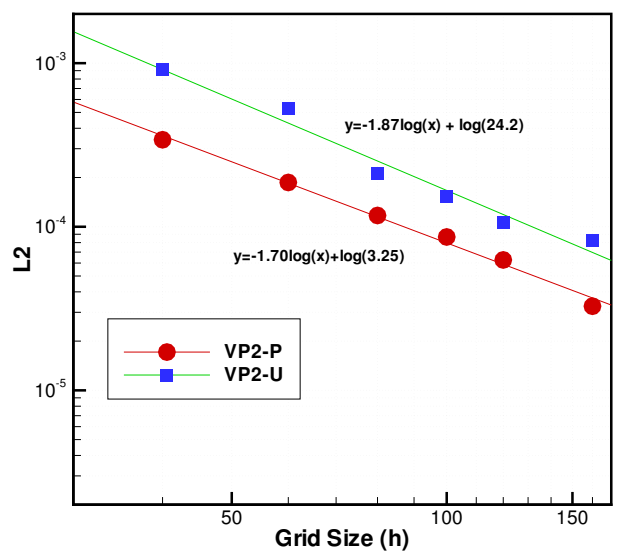

Figure 16. L2-norm of pressure and u-velocity at $\mathbf{T}=60$.

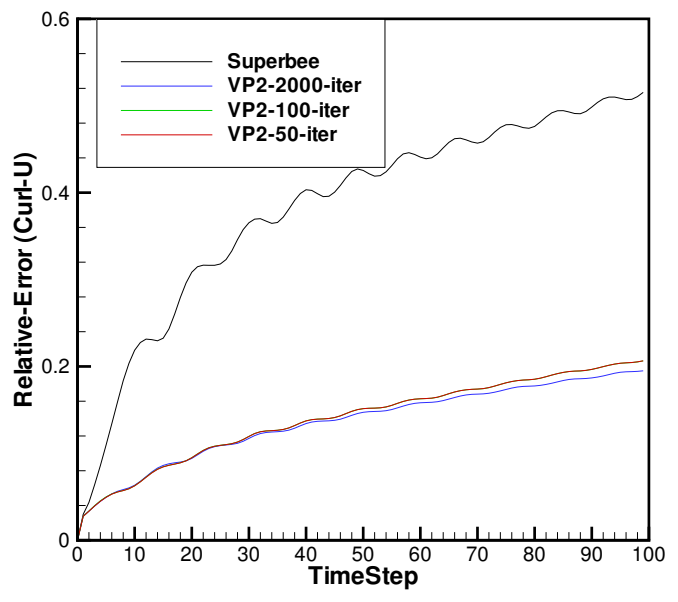

Figure 15. Normalized L2-Norm of $\omega(80 \times 80)$ for second order schemes: Pure Hancock with Superbee limiter and Hancock with vorticity preserving (VP2) scheme at various sub-iterations steps.

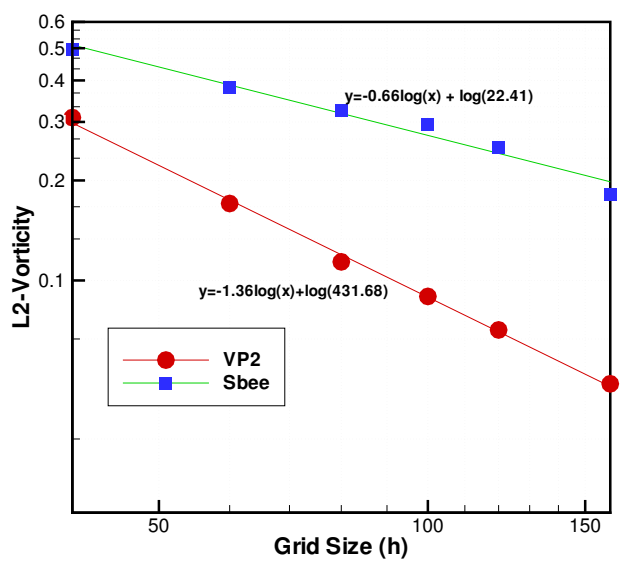

Figure 17. L2-norm of $\omega$ at $\mathbf{T}=60$. Note that VP2 is twice more accurate than conventional second order Hancock-Superbee. 